\title{
Localization of a Single Binding Site for Immunoglobulin Light Chains on Human Tamm-Horsfall Glycoprotein
}

\author{
Zhi-Qiang Huang ${ }^{\star \ddagger}$ and Paul W. Sanders ${ }^{\star \ddagger}$ \\ *Department of Veterans Affairs Medical Center, Birmingham, Alabama 35233; and ${ }^{\ddagger}$ Nephrology Research and Training Center, \\ Comprehensive Cancer Center, and Cell Adhesion and Matrix Research Center Division of Nephrology, Department of Medicine and \\ Department of Physiology \& Biophysics, University of Alabama at Birmingham, Birmingham, Alabama 35294-0007
}

\begin{abstract}
Cast nephropathy is a severe complication of multiple myeloma. Binding of filtered monoclonal light chains (LC) with Tamm-Horsfall glycoprotein (THP) triggers heterotypic aggregation of these two proteins to form casts in the distal nephron of the kidney. To localize the LC binding site on THP, human THP was deglycosylated and underwent limited trypsin digestion in the presence or absence of a nephrotoxic LC known to bind THP. A 29.6-kD band was protected from trypsin digestion by the addition of $\mathrm{LC}$. $\mathrm{NH}_{2}$-terminal amino acid sequence and amino acid analyses revealed this band was located between the 6 th and 287 th amino acid residues of THP. Six peptides located within this 29.6-kD fragment were synthesized and used as potential inhibitors of binding or aggregation of five different nephrotoxic LCs with THP. Peptide AHWSGHCCL (from amino acid 225 to 233 ) completely inhibited binding and aggregation of these proteins. Optimal inhibition required a cystine residue in this peptide. Truncation experiments demonstrated the entire sequence was necessary for ideal inhibition and the histidine residue explained the effects of $\mathrm{pH}$ on binding. These studies provided a basis for further study of LC-THP interaction and a potential approach toward the prevention of cast nephropathy. (J. Clin. Invest. 1997. 99: 732-736.) Key words: Bence Jones protein - cast nephropathy - acute renal failure - multiple myeloma • myeloma kidney
\end{abstract}

\section{Introduction}

Tamm and Horsfall described in 1950 a unique urinary protein that inhibited viral hemagglutination in vitro (1). This TammHorsfall glycoprotein (THP), ${ }^{1}$ which has since been characterized as a 616-amino acid glycoprotein (2), is synthesized specifically by cells of the thick ascending limb of the loop of Henle $(3,4)$. About $30 \%$ of the molecular weight of THP is

Address correspondence to Paul W. Sanders, Division of Nephrology/Department of Medicine, 642 Lyons-Harrison Research Building, University of Alabama at Birmingham, Birmingham, AL 35294-0007. Phone: 205-934-3589; FAX: 205-975-6288; E-mail: paul_sanders@ nrtc.dom.uab.edu

Received for publication 3 June 1996 and accepted in revised form 4 December 1996.

1. Abbreviations used in this paper: $\mathrm{LC}$, monoclonal immunoglobulin light chains; LCBP, light chain binding peptide; PBCL, complementary peptide to the LCBP; THP, Tamm-Horsfall glycoprotein.

The Journal of Clinical Investigation

Volume 99, Number 4, February 1997, 732-736 carbohydrate (5). Although the physiologic significance of THP remains undefined, this protein plays an integral role in the pathologic condition known as cast nephropathy. Cast nephropathy, or "myeloma kidney," is a common complication of multiple myeloma. Binding and subsequent coaggregation of filtered immunoglobulin light chains (LCs) with THP obstruct flow through the renal tubule and result in renal failure $(6,7)$. Both $\kappa$ and $\lambda$ LCs share a common binding site on THP, although different nephrotoxic LCs possess different affinities for THP (6). Identifying and characterizing the protein-protein interaction between LCs and THP allow development of new therapeutic strategies to decrease incidence of this complication. The current experiments were designed to determine the light chain binding site on human THP.

\section{Methods}

Protein and peptide preparations. THP was purified from urine of a normal male in standard fashion (8) using original method of Tamm and Horsfall (1). THP was precipitated by using $0.64 \mathrm{M} \mathrm{NaCl}$, dialyzed against water adequately and lyophilized. Five LCs, mic (к isotype), and don, bol, wil, and hor (all $\lambda$ isotypes), were used in this study. These LCs were purified from urine of patients who had multiple myeloma and renal failure by precipitation using $70 \%$ ammonium sulfate, followed by ion exchange chromatography (9). Purified LCs were dialyzed against water adequately and lyophilized. The isoelectric point was 7.7 for mic, 5.7 for $\mathrm{don}$, and 5.1 for $\mathrm{bol}$. Peptides used in these studies were synthesized by the protein synthesis core facility of the Comprehensive Cancer Center of the University of Alabama at Birmingham and by Research Genetics Inc. (Huntsville, AL). Peptides were kept lyophilized at $-20^{\circ} \mathrm{C}$ until use. To determine the role of cystine in the binding interaction, some peptides containing cysteine residues were diluted to $10 \mathrm{mg} / \mathrm{ml}$ in $0.1 \%$ trifluoroacetic acid (Sigma Chemical Co., St Louis, MO), and then further purified using reverse-phase high performance liquid chromatography with a $\mathrm{C}_{18}$ preparative column (Novopack; Waters Processing Supplies, Inc., Marlborough, MA). The flow rate of mobile phase was $1 \mathrm{ml} / \mathrm{min}$. An acetonitrile gradient from 0 to $100 \%$, which was controlled using a two-pump delivery system (501 pump solvent delivery system; Waters Processing Supplies, Inc.), separated the peptides. The molecular weight of the eluted peptides was determined by mass spectrometry provided by a core facility of the Comprehensive Cancer Center. Ellman's reagent (Caymen Chemical Co., Inc., Ann Arbor, MI) was used to detect free sulfhydryl groups of these peptides.

One peptide, the light chain binding peptide (LCBP), was used as a template to design a complementary peptide according to Weigent et al. (10). The complementary peptide (PBCL) was constructed based upon the RNA sequence that was complementary to the RNA strand encoding the amino acid sequence of LCBP. The hydropathy profile of each amino acid of the complementary and template peptides was determined using computer software (MacVector; Eastman Kodak Co., New Haven, CT).

Trypsin protection experiment. Purified THP, $10 \mathrm{mg} / \mathrm{ml}$, was suspended in $1 \%$ SDS and boiled for 5 min. Denatured THP was then mixed in sodium phosphate buffer, $\mathrm{pH} 8.6$, containing $160 \mathrm{mM}$ so- 
dium phosphate, $5 \mathrm{mM}$ 1,10-phenanthroline, $0.3 \%$ Triton X-100, and $12.5 \mathrm{U} / \mathrm{ml} \mathrm{N}$-glycosidase F (PNGase F; Boehringer Mannheim Biochemicals, Indianapolis, IN). The final concentration of THP was 1.5 $\mathrm{mg} / \mathrm{ml}$. The mixture was incubated at $37^{\circ} \mathrm{C}$ for $18 \mathrm{~h}$. Deglycosylated THP was concentrated to $1 / 3$ of the original volume using a microconcentrator (Centricon 30; Amicon Inc., W.R. Grace \& Co., Beverly, MA), then diluted 20 times using $0.05 \mathrm{M}$ Tris $/ \mathrm{HCl}, \mathrm{pH} 8.6$, and again concentrated. The final concentration of deglycosylated THP was $\sim 6$ $\mathrm{mg} / \mathrm{ml}$. Trypsin, $0.12 \mathrm{mg} / \mathrm{ml}$, was mixed with deglycosylated THP and incubated at $37^{\circ} \mathrm{C}$ for $22 \mathrm{~h}$ in the presence or absence of an equimolar amount of mic. The products of trypsin digestion were electrophoresed on a $7.5 \%$ SDS polyacrylamide gel, and then transferred to a polyvinylidene fluoride membrane (Millipore Corp., Bedford, MA). After blocking with 5\% milk in PBS and washing with $0.05 \%$ Tween 20 in PBS, the membrane was incubated with rabbit anti-human THP antiserum (Biomedical Technologies, Inc., Stoughton, MA), 1:1,000 dilution in PBS, followed by incubation with horseradish peroxidaseconjugated goat anti-rabbit IgG (Bio-Rad Laboratories, Melville, $\mathrm{NY}$ ). The membrane was washed again and developed using peroxi-

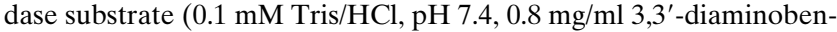
zidine, $0.01 \%$ hydrogen peroxide). $\mathrm{NH}_{2}$-terminal amino acid sequence determination (10 cycles) and amino acid analysis of digestion products were performed by Dr. William Lane at the Biological Laboratories, Harvard University, Cambridge, MA. The results were compared with the known sequence of human THP (2).

Binding analysis and peptide blocking studies. Binding of LCs with THP was analyzed using a solid-phase enzyme-linked immunoassay (6). To observe if a peptide fragment blocked the binding of LCs and THP, a competition study was used. Wells of microplates were coated with each LC, $0.2 \mathrm{mM}$ in PBS, and incubated overnight at room temperature. After washing with $0.05 \%$ Tween 20 in PBS and blocking with $1 \%$ bovine serum albumin in PBS, THP, $0.19 \mu \mathrm{M}$ in $2 \%$ Tween 20 in PBS, was added to the wells simultaneously with each of the different peptide constructs. The concentration of peptides in this study was $0-7.5 \mathrm{mM}$. The mixtures were incubated for $30 \mathrm{~min}$ at room temperature. A mouse monoclonal $\mathrm{IgG}_{1} \kappa$, which was raised in our laboratory and found to be directed specifically against the carbohydrate component of human THP, was added, followed by horseradish per-

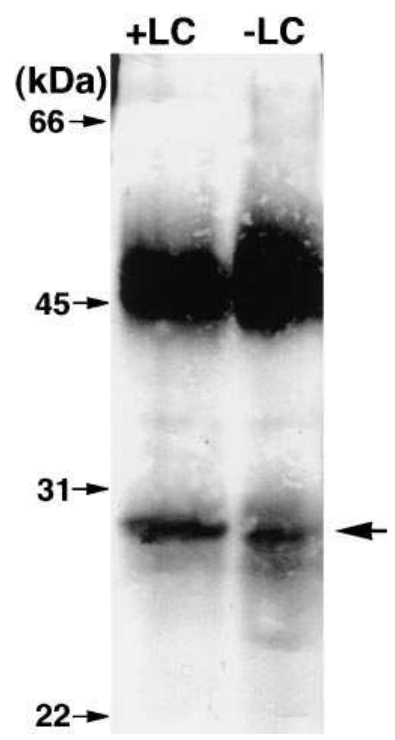

Figure 1. Results of limited trypsin digestion of deglycosylated THP in the presence $(+L C)$ or absence $(-L C)$ of light chain. Trypsin digestion products were separated using SDS-PAGE, and then transferred to polyvinylidene flouride membrane and reacted with rabbit anti-human THP antibody. The membrane was then reacted with horseradish peroxidase-conjugated goat anti-rabbit IgG. In the presence of light chain (left lane), a 29.6-kD band (arrow) was protected by mic from digestion by trypsin.

oxidase-conjugated goat anti-mouse IgG (Bio-Rad Laboratories). Azino-bis(3-ethylbenzthiazoline-6-sulfonic acid), $2.5 \mathrm{mg} / \mathrm{ml}$ in citratephosphate buffer, $\mathrm{pH} 4.2$, was used as substrate for horseradish peroxidase. In these experiments, $100 \%$ binding of THP represented a maximum of $20 \mathrm{pmol} / \mathrm{well}$.

Turbidity measurement. Light-scattering analysis $(6,8)$ was used to observe the heterotypic aggregation of LCs and THP in solution. The turbidity of PBS containing THP, $5 \mu \mathrm{M}$, was monitored at 488 $\mathrm{nm}$ for $20 \mathrm{~min}$ using a spectrofluorometer (M-series; Photon Technology International, Inc., South Brunswick, NJ) after adding each light chain, $2.7 \mathrm{mM}$. To determine competitive inhibition of aggregation of LCs with THP, test peptides were added simultaneously with the LCs.

Statistics. All data were expressed as mean \pm standard error. A linear regression or single exponential decay with offset was used to

Table I. Effect of Synthetic Peptides in Preventing Binding between LCs and THP. The IC ${ }_{50}$ Was Calculated using Data from the Binding Analysis Studies

\begin{tabular}{|c|c|c|c|c|}
\hline \multirow[b]{2}{*}{ Position on THP } & \multirow[b]{2}{*}{ Peptide sequence } & \multicolumn{3}{|c|}{$\mathrm{IC}_{50}(\mathrm{mM})$} \\
\hline & & mic & don & bol \\
\hline $72-81$ & EGFRLSPGLG & NE & $\mathrm{NE}$ & $\mathrm{NE}$ \\
\hline $118-120$ & RGD & NE & $\mathrm{NE}$ & $\mathrm{NE}$ \\
\hline $127-130$ & SPGS & $\mathrm{NE}$ & NE & NE \\
\hline $157-169$ & DEYWRSTEYGEGY & NE & NE & NE \\
\hline $176-186$ & RGWYRFVGQGG & $\mathrm{NE}$ & $\mathrm{NE}$ & $\mathrm{NE}$ \\
\hline $225-233$ & AHWSGHCCL & $1.5 \pm 0.7$ & $0.6 \pm 0.1$ & $0.5 \pm 0.1$ \\
\hline \multicolumn{5}{|c|}{ Modifications of LCBP (peptide 225-223) } \\
\hline & AHWSGHCCL (D-amino acids) & $0.7 \pm 0.3$ & $0.6 \pm 0.2$ & $0.4 \pm 0.2$ \\
\hline & AHWSGSCCL & $0.6 \pm 0.04$ & $0.4 \pm 0.05$ & $0.5 \pm 0.05$ \\
\hline & AHWSGHSSL & $8.2 \pm 2.6^{*}$ & $9.8 \pm 1.2^{*}$ & $7.4 \pm 0.7$ \\
\hline & AHWSGHC* $\mathrm{C} * \mathrm{~L}$ & NE & NE & NE \\
\hline & GHCCL & $6.5 \pm 1.2$ & $5.1 \pm 0.7$ & $4.0 \pm 1.5$ \\
\hline & GSCCL & $4.2 \pm 0.2$ & $3.5 \pm 0.5$ & $4.0 \pm 0.3$ \\
\hline & AHWSG & $\mathrm{NE}$ & NE & $\mathrm{NE}$ \\
\hline & AHWSGHG & NE & $\mathrm{NE}$ & $\mathrm{NE}$ \\
\hline \multicolumn{5}{|c|}{ Complementary peptides } \\
\hline & QAAVAAPVR & $0.5 \pm 0.04$ & $0.2 \pm 0.03$ & $0.2 \pm 0.03$ \\
\hline & QAAVA & $2.6 \pm 1.4$ & $2.3 \pm 0.3$ & $0.6 \pm 0.3$ \\
\hline
\end{tabular}

$\mathrm{C}^{*}$, cysteines that have covalent modifications on the sulfhydryl group; NE, no effect; *extrapolated values. 


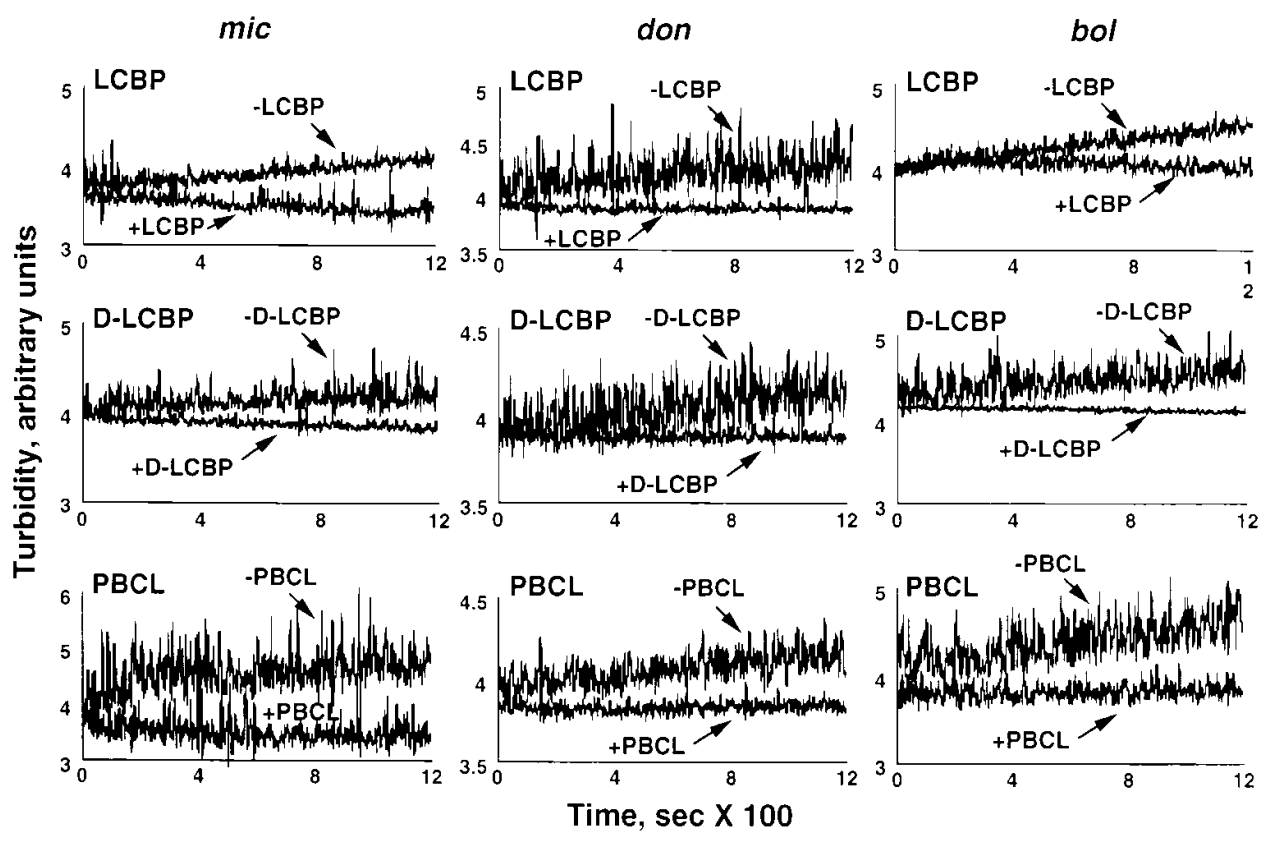

Figure 2. Aggregation rates of LCs, $2.7 \mathrm{mM}$, to THP, $5 \mu \mathrm{M}$, in the presence of LCBP, LCBP synthesized with $\mathrm{D}$ amino acids (D-LCBP), and PBCL. LCBP and D-LCBP, 100 $\mu \mathrm{M}$, and PBCL, $15 \mu \mathrm{M}$, were added simultaneously with THP into PBS containing LCs. All three peptides completely inhibited heterotypic aggregation of LCs to THP. calculate the $\mathrm{IC}_{50}$ of the inhibitory peptides. Differences between data sets were determined using unpaired $t$ test, with significance set at $5 \%$.

\section{Results}

One band located at $29.6 \mathrm{kD}$ was found to be protected by mic from digestion by trypsin (Fig. 1). $\mathrm{NH}_{2}$-terminal amino acid sequence analysis and amino acid analysis showed this band was located between the 6 th and $287 \mathrm{th}$ amino acid residues of THP. This region of THP was examined using Kyte-Doolittle hydrophobicity data to determine those segments that had a high probability to be on the surface of the molecule, allowing potential interaction with light chains. Six peptides predicted to be on the surface of the region between the 6th and 287th amino acid residues were synthesized (Table I). Only one of these six synthesized peptides, which was identical to the amino acid sequence from 225 to 233 of human THP and designated LCBP, blocked the binding of LCs with THP in a concentration-dependent manner. The ability to inhibit binding differed among the three LCs (Table I) and was consistent with the affinities of these LCs for THP (6). Mic possessed the highest affinity for THP (6), while bol had the lowest affinity. This LCBP also inhibited binding of wil and hor to THP in a concentration-dependent fashion. In turbidity studies, this same peptide (LCBP) inhibited heterotypic aggregation of LCs with THP (Fig. 2).

Seven peptide fragments were synthesized, representing truncation or replacement of amino acid residues of the LCBP (Table I). Peptides AHWSG and AHWSGHG did not inhibit binding between LCs and THP. Replacement of either or both cysteines with serine decreased or eliminated this inhibitory effect. Furthermore, peptide AHWSGHC*C*L, which was synthesized using acetamidomethyl-cysteines (represented by $\mathrm{C}^{*}$ ), had no effect on the binding of LCs to THP. Removal of AHWS residues from the $\mathrm{NH}_{2}$-terminal of the LCBP reduced the inhibitory capability of the peptide; inhibition remained incomplete even at high doses of peptide. Inhibitory activity was partially restored by changing $\mathrm{H}$ to $\mathrm{S}$ to produce GSCCL. AHWSGSCCL produced greater $(P<0.025)$ inhibition of the interaction between mic and THP than AHWSGHCCL (Table I). Holo-LCBP synthesized with D amino acids (termed D-LCBP) inhibited binding (Table I) and heterotypic aggregation of LCs with THP in turbidity studies (Fig. 2).

GHCCL, holo-LCBP, and D-LCBP were further subjected to HPLC analysis. Similar patterns with multiple peaks were found with all three peptides. The major peaks represented single molecules by mass spectrometry. Only these peaks, which did not possess free sulfhydryl groups demonstrated using Ellman's reagent, inhibited the binding interaction of LCs with THP.

Complementary peptide PBCL (QAAVAAPVR) also inhibited binding of LCs to THP in a dose-dependent manner (Fig. 3). This peptide also inhibited binding of wil and hor to

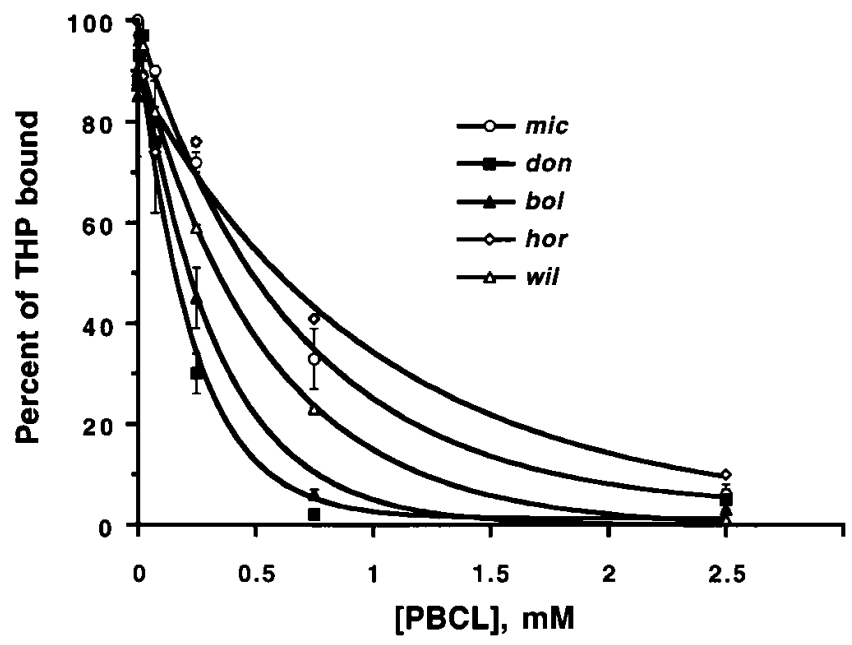

Figure 3. Inhibition of the binding of THP to wells containing five different LCs. PBCL effectively prevented binding in a dose-dependent manner. 

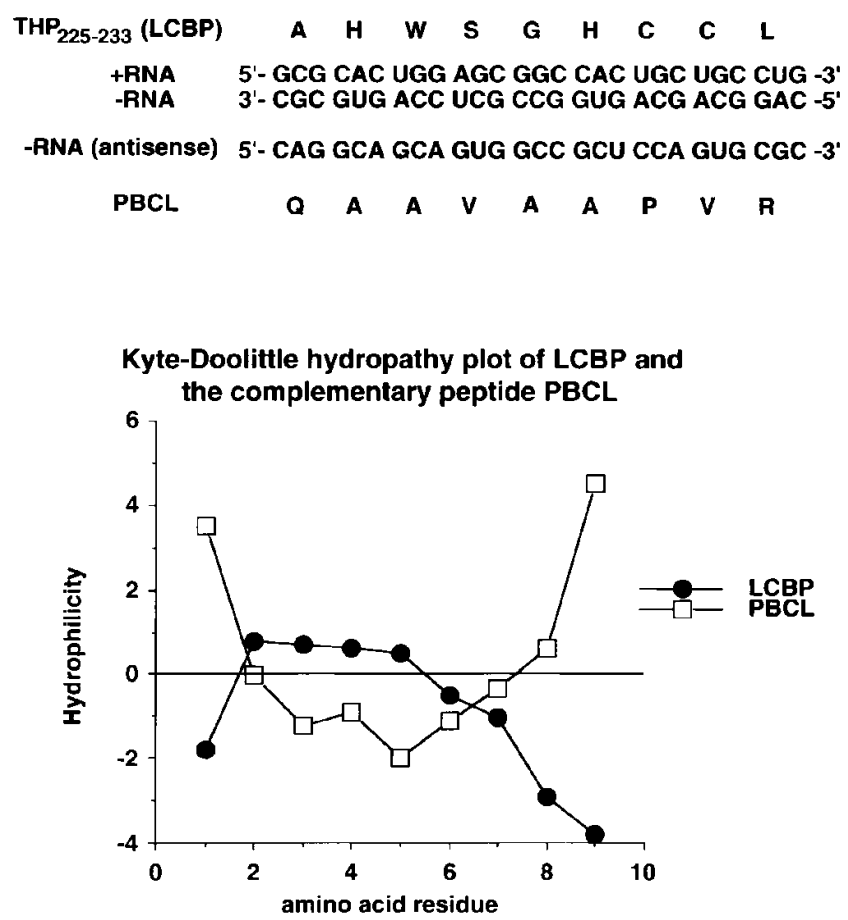

Figure 4. Sequence information and hydropathy plots of LCBP and the complementary peptide PBCL. PBCL was designed according to the complementary sequence of DNA encoding LCBP. These two peptides have inverted patterns of hydrophilicity.

THP, with $\mathrm{IC}_{50}$ of $0.4 \pm 0.03$ and $0.6 \pm 0.06 \mathrm{mM}$, respectively. Hydropathy plots showed PBCL possessed a pattern opposite that of LCBP (Fig. 4). To test further the interaction between the complementary peptide and THP, a solid-phase binding assay was performed. As expected, THP bound to peptide PBCL in a dose-dependent manner (Fig. 5). PBCL also completely inhibited aggregation of LCs to THP (Fig. 2). Finally, truncation of PBCL to produce QAAVA resulted in a peptide that was complementary to GHCCL. This peptide also inhibited binding of LCs to THP, although the $\mathrm{IC}_{50}$ was higher and did not completely prevent binding of mic to THP.

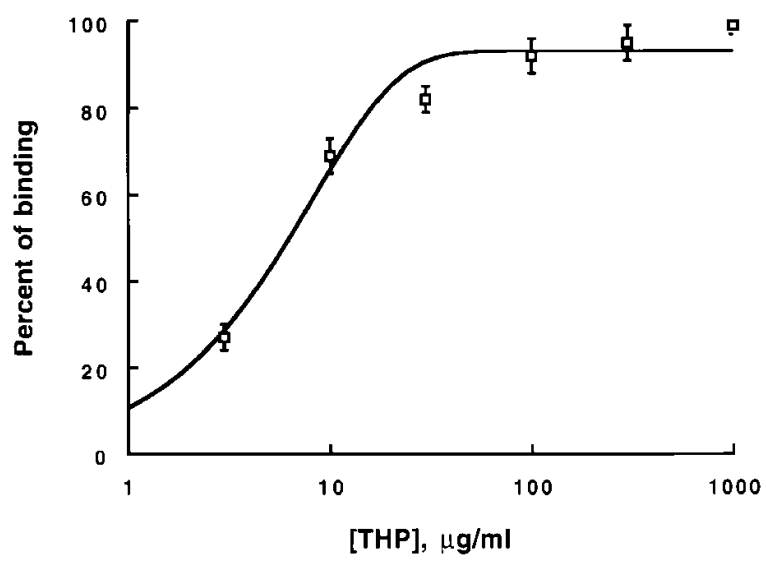

Figure 5. Binding assay using PBCL, $10 \mu \mathrm{M}$, which was preadsorbed to wells of microtiter plates, and THP. PBCL bound THP, but THP did not bind to wells containing albumin only. The molar concentration of THP, $1,000 \mu \mathrm{g} / \mathrm{ml}$, is $10 \mu \mathrm{M}$.

\section{Discussion}

THP and LCs are the two major components of casts that obstruct the nephron and produce cast nephropathy or "myeloma kidney." Our previous studies showed that LCs of both isotypes bound to a common site on the peptide backbone of THP, although with different affinities. This noncovalent binding interaction triggered subsequent heterotypic aggregation of LCs with THP (6). These biochemical interactions between LCs and THP form the basic mechanism of cast formation in vivo $(6,7)$. In the current study, limited trypsin digestion of THP with the simultaneous addition of a light chain known to bind THP localized the binding site of LC on THP to a $29.6-\mathrm{kD}$ peptide fragment between amino acids 6 and 287. One synthesized peptide corresponding to amino acids 225-233 (AHWSGHCCL), designated LCBP, inhibited binding and aggregation of THP with five different light chains. The cystine residue was important for optimum inhibition. The $\mathrm{NH}_{2}$-terminal portion of this peptide, AHWSG, did not inhibit the binding between LCs and THP. However, removal of AHWS from the LCBP caused an obvious decline in inhibiting capability (Table I). Thus, the entire 9-amino acid residues appeared to form the light chain binding domain. Finally, a complementary peptide, PBCL, which had a hydropathic profile opposite that of LCBP, bound THP and completely inhibited THP-LC interactions. These combined studies demonstrated the single binding site on THP for LC.

The current studies also complemented our previous experiments that examined the binding interaction between LCs and THP. Pretreatment of THP with a variety of reducing agents inhibited LC-THP interactions (6). This finding was consistent with the observation that the intramolecular disulfide bond between cysteine 231 and 232 provided an ideal conformation to produce optimal inhibition. Without the cystine residue, the binding interaction was much less effective or disappeared. The inhibitory effect of binding between LCs and THP by synthetic LCBP containing a cystine residue indicates the effect of reducing agents was due to cleaving the disulfide bond between cysteine 231 and 232 .

We also determined that varying $\mathrm{pH}$ between 5 and 8.5 , which alters the charge of the imidazole ring on histidine, modified LC-THP interactions (11). In the current study, replacement of the negatively charged histidine with serine enhanced the capability of the peptide to inhibit binding of mic, but not don and bol, to THP at neutral pH (Table I). Thus, the histidine residue may explain the effect of $\mathrm{pH}$ in altering the binding interaction between THP and some of these proteins (11). Ambient $\mathrm{pH}$ may also affect the charges of side chains of certain amino acid residues on LCs, causing changes in binding affinity.

To characterize further the LC-THP interaction, these studies also employed a recently described method that designs novel peptides based on the genetic code of the binding site for ligands on peptide hormone receptors (10). This molecular recognition theory proposed by Weigent et al. (10) suggests that a peptide synthesized according to the DNA sequence complementary to the DNA sequence encoding another peptide will interact with that peptide. In our experiments, PBCL was designed according to the complementary sequence of DNA encoding LCBP (Fig. 3) and was shown to bind THP (Fig. 4). This peptide completely inhibited LC-THP interactions and provided further evidence that the LCBP defined the 
LC binding site of THP. Because the hydropathy plot of PBCL was opposite that of the LC binding peptide (Fig. 3), we have proposed that cast-forming LCs contain peptide sequences that possessed this same inverted hydropathic profile to allow interaction with the LC binding site. Thus, a defined linear hydropathic profile and not a specific amino acid sequence per se may be the most important determinant in the interaction with THP. The results using PBCL are extremely useful for further study of the THP binding site on LCs, and may facilitate the definition of those sequences on LCs that interact with THP.

In summary, the light chain binding site on THP was shown to be localized to a linear sequence of nine amino acids: $\mathrm{AH}$ WSGHCCL. Immunohistochemistry studies of kidney biopsy specimens from patients with cast nephropathy have shown that THP is an integral part of these casts (12). Modification of THP by colchicine inhibited aggregation with LCs in human subjects (11), and prevented cast formation in a rat model of cast nephropathy (7), thus further showing an important role for THP in this process. Identification of the light chain binding site on THP will help to produce strategies that inhibit interaction of LCs with THP and potentially prevent a severe renal complication of multiple myeloma.

\section{Acknowledgments}

The authors thank Ms. Melinda McMorris for her excellent technical assistance and the Media Service of the Birmingham Veterans Affairs Medical Center for the photography and illustrations.

This work was supported by the Office of Research and Develop- ment, Medical Research Service, Department of Veterans Affairs, and a grant from the American Cancer Society.

\section{References}

1. Tamm, I., and F.L. Horsfall, Jr. 1950. Characterization and separation of an inhibitor of viral hemagglutination present in urine. Proc. Soc. Exp. Biol. Med. 74:108-114.

2. Pennica, D., W.J. Kohr, W.-J. Kuang, D. Glaister, B.B. Aggarwal, E.Y. Chen, and D.V. Goeddel. 1987. Identification of human uromodulin as the Tamm-Horsfall urinary glycoprotein. Science (Wash. DC). 236:83-88.

3. Bachmann, S., R. Metzger, and B. Bunnemann. 1990. Tamm-Horsfall protein-mRNA synthesis is localized to the thick ascending limb of Henle's loop in rat kidney. Histochemistry. 94:517-523.

4. Hoyer, J.R., and M.W. Seiler. 1979. Pathophysiology of Tamm-Horsfall protein. Kidney Int. 16:279-289.

5. Fletcher, A.P., A. Neuberger, and W.A. Ratcliffe. 1970. Tamm-Horsfall urinary glycoprotein. Biochem. J. 120:417-424.

6. Huang, Z.-Q., K.A. Kirk, K.G. Connelly, and P.W. Sanders. 1993. Bence Jones proteins bind to a common peptide segment of Tamm-Horsfall glycoprotein to promote heterotypic aggregation. J. Clin. Invest. 92:2975-2983.

7. Sanders, P.W., and B.B. Booker. 1992. Pathobiology of cast nephropathy from human Bence Jones proteins. J. Clin. Invest. 89:630-639.

8. Sanders, P.W., B.B. Booker, J.B. Bishop, and H.C. Cheung. 1990. Mechanisms of intranephronal proteinaceous cast formation by low molecular weight proteins. J. Clin. Invest. 85:570-576.

9. Sanders, P.W., G.A. Herrera, and J.H. Galla. 1987. Human Bence Jones protein toxicity in rat proximal tubule epithelium in vivo. Kidney Int. 32:851861.

10. Weigent, D.A., B.L. Clarke, and J.E. Blalock. 1994. Peptide design using a genetically patterned binary code: growth hormone-releasing hormone as a model. Immunomethods. 5:91-97.

11. Huang, Z.-Q., and P.W. Sanders. 1995. Biochemical interaction of Tamm-Horsfall glycoprotein with Ig light chains. Lab. Invest. 73:810-817.

12. Start, D.A., F.G. Silva, L.D. Davis, V. D’Agati, and C.L. Pirani. 1988. Myeloma cast nephropathy: immunohistochemical and lectin studies. Mod. Pathol. 1:336-347. 